\title{
Introduction to Special Section on JPEG 2000 Digital Imaging
}

\author{
Guest Editors: Touradj Ebrahimi and Daniele D. Giusto
}

In the past 20 years, the industry that deals with digital imaging has taken advantage of two major technological improvements, which deeply changed the way it does business: common availability of numerical image data and the Internet. A third innovation was recently introduced and is due to become a commercial reality in a short time: mobile imaging.

Sector analysts predict that the sales of mobile phones with embedded cameras will increase by $500 \%$ yearly, moving from the 20 millions of units in 2002 to the 150 millions in 2005, thus overtaking the number of digital cameras. Such increase, which finds no equivalent in the technological industry, will in a few years enable hundreds of millions of people to have handy devices for the acquisition and transmission of digital images.

This is just one of many aspects showing excellent expectations and possibilities for the standard that defines the internationally recognized specifications for digital image exchange. Market surveys from several international institutions have shown that the JPEG standard is widely recognized and the new JPEG 2000 is due to gain an important role and eventually replace its predecessor. This new standard was released by the ISO/SC29/WG01 (JPEG) international standardization committee in December 2000, and was subsequently published by ISO in the spring of the following year. JPEG 2000 is meant to be a novel tool for visual information distribution with multiple features in a multipurpose applications scenario and will replace the previous standard, released almost ten years ago and unable to meet future market requirements.

As an example, the infusion of digital cameras has grown to be so substantial in the past year that the number of units sold actually overtook that of classical photographic cameras. Furthermore, research and improvement on digital memories (flashcards can go up to $1 \mathrm{~GB}$ at the moment) make possible on-board storage of hundreds, if not thousands, of images. At the same time, the power of mobile-computing processors is increasing exponentially, providing computational capabilities comparable to those of two-year old desktop computers. Another interesting fact is that surveys on Internet applications show that the JPEG file format is by far the most used (by a factor of more than 10) among other multimedia formats, such as video MPEG and audio MP3. All these facts together confirm what the ISO/JPEG committee predicted in the second half of the nineties, when it started to plan its activities towards the definition of a new standard in the field of digital imaging, which would result in a state-of-the-art multipurpose tool for visual information exchange.

In 1997, during the Sydney JPEG committee meeting, a Call for Proposals was released that would result, by the end of 2000 at the New Orleans meeting, in the definition of the new standard, named JPEG 2000. Its new architecture is based on the betterperforming Wavelet Transform, instead of the previous Discrete Cosine Transform, thus enabling scalability, a crucial feature for multipurpose applications and hardware support.

JPEG 2000 part 1 is the base version of the standard, where common codec architecture and digital image format are defined. In 2001 major parts of the standards were settled (Motion JPEG 2000, reference software, file format, for example), and in 2002 the JPEG committee promoted several actions to design and to develop additional new features for several applications:

1) Image transmission security (JPEG 2000 part 8 - JPSEC). Security issues, such as authentication, data integrity, intellectual property protection and conditional data access among others, are important aspects in many applications targeted by JPEG 2000. The committee is working to produce tools and solutions to such issues, in the form of development specifications.

2) Interactivity (JPEG 2000 part 9 - JPIP). This part is committed to the definition of techniques for designing the humanmachine interface in order to fully exploit the standard's capabilities both for local and remote applications. Interactivity is indeed a key aspect in all multimedia applications and requires the definition of rules and syntax for the exchange of information. The purpose of JPIP is to provide project and working specifications for flexible and interactive applications.

3) Volumetric data representation (JPEG 2000 part 10 - JP3D). The use of volumetric is common in several fields, such as medicine, biology, geology and astronomy. Such data need to be stored and processed with high efficiency and quality. Currently, a standard 3-dimensioinal data format is not provided, a good reason for extending JPEG 2000 capabilities given its superior performance in image compression and quality. JP3D is focused on the definition of representation and coding techniques for 3D data, either in their original or transformed form.

4) Wireless image communication (JPEG 2000 part 11 - JPWL). An implementation of the standard for wireless applications requires specific features and solutions, in order to take advantage of each communication system's characteristics, such as 3rd generation mobile or Bluetooth terminals and wireless LANs. The purpose of JPWL is to extend the standard to these fields to fully exploit its possibilities.

At the moment, the activity is in the study and pre-experimentation stage, since the working drafts for each subject are being defined. Ongoing activities are related to the standardization and development of novel systems and applications, such as all encompassing personal communications, where each user is provided with network-connected mobile-computing devices for complete and global interaction. Such scenarios create several issues, such as scalability for different user devices, wireless transmission reliability, content protection, user interaction capability and the possibility of content editing from the terminal device. All these aspects are being considered and studied by the JPEG committee. 
The idea for this Special Section arose when organizing the First International Workshop on JPEG 2000, held in Lugano, Switzerland, on July 8, 2003, a forum to promote and exchange knowledge and experience related to the new standard. We launched a call early this year, to collect papers from industry and academic researchers, with the intent of offering a view on new products and scenarios related to JPEG 2000.

In this Special Section readers will find papers analyzing performance of the standard, describing hardware and software implementations of the standard, as well as a view of some activities in developing new parts of JPEG 2000.

In particular, the first part of this Special Section (devoted to implementations and applications) opens with a paper by Battiato et al. dealing with different implementations and performance evaluation of the standard. Schumacher presents an efficient hardware implementation of JPEG 2000, especially for FPGA platforms. The paper by Foessel et al. describes Motion JPEG 2000, evaluates its performance against MPEG, and illustrates hardware implementations. In the next paper, Wu and Deng present a content-aware stream authentication scheme in Motion JPEG 2000 video distribution over lossy networks. Part 6 of the standard (Compound Image File Format) is addressed by Jung and Seiler, who analyze JPEG 2000 performance on document imaging. The following, two papers (by Nguyen et al. and by Signoroni et al.) deal with region of interest (ROI) coding, a new powerful tool for improving picture compression without losing quality where needed. This first group of articles is completed by three papers dealing with applications: Su and Kuo present a scheme for hiding data in a coded picture, Bilgin et al. describe how the standard could be used in representation and compression of single dimensional signals, and Minelli and De Polo illustrate JPEG 2000 potentials in the fast emerging field of mobile imaging.

The second part of this Special Section deals with advanced parts of JPEG 2000, still under definition at this time, and offers papers on JPSEC (by Sadourny and Conan, describing data encryption), JPIP (by Colyer and Clark, who illustrate the JPEG 2000 framework for interactive applications), and JPWL. Wireless transmission is the main subject of the last four papers, where Nicholson et al. describe error control strategies in JPEG 2000, Frescura et al. illustrate JPEG 2000 and Motion JPEG 2000 data transmission in WiFi environments, and Grangetto et al. depict data structure and transmission strategy. The paper by Atzori on unequal power allocation for efficient and robust transmission closes the Special Section.

As a final remark, this section does not include any tutorials on JPEG 2000, since many excellent relevant articles may be found in the literature. For instance, the interested readers could find brief, yet complete, information in [1,2], while in depth descriptions can be found in [3].

\section{ACKNOWLEDGMENT}

The Guest Editors thank the European Commission in support of this initiative through the IST-2001-34096-2KAN project (Work Package 10: Dissemination and Exploitation Planning).

\section{REFERENCES}

[1] M.Rabbani and R.Joshi, "An overview of the JPEG 2000 still image compression standard," Image Communication, vol. 17, no. 1, pp. 3-48, 2002.

[2] C.Christopoulos, A.Skodras, and T.Ebrahimi, "The JPEG 2000 still image coding system: An overview," IEEE Transaction on Consumer Electronics, vol. 46, no. 4, pp. 1103-1127, 2000.

[3] D.S.Taubman and M.W.Marcellin, JPEG 2000: Image compression fundamentals, standards and practice, Kluwer Academic Publishers: Norwell, 2002.

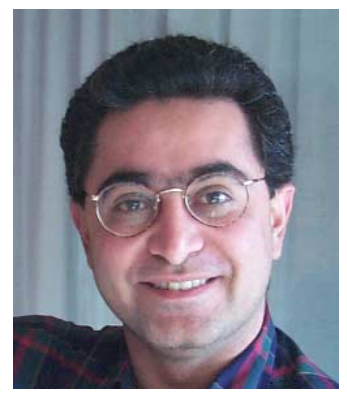

Touradj Ebrahimi received his M.S. degree in electrical engineering and his Ph.D. degree in digital image processing from EPFL, Switzerland, in 1989 and 1992. From 1992 to 1994 he worked with Sony and AT\&T Bell Labs. In 1994 he joined EPFL where he is Professor of Image Processing and leads the Visual Information Processing Group. He is responsible of a number of EU and national research projects, and head of the Swiss delegation within the ISO JPEG and MPEG standardization committees. His research interests are in media technology and interactivity.

Dr. Ebrahimi is an associate editor of IEEE Trans. Multimedia, SPIE Optical Engineering and EURASIP Applied Signal Processing journals, and an area editor of Image Communication journal; he was associate editor of IEEE Trans. Image Processing until 2003, as well as a guest editor for a number of special issues of international journals. He authored a hundred technical papers, holds a dozen patents, and coauthored the MPEG-4 Book (Prentice-Hall, 2002).

Dr.Ebrahimi is the recipient of the 2000 IEEE Chester Sall best paper award, two Swiss awards and two ISO certificates for outstanding contributions to JPEG2000 and MPEG-4 standardization. He is a fellow of SPIE.

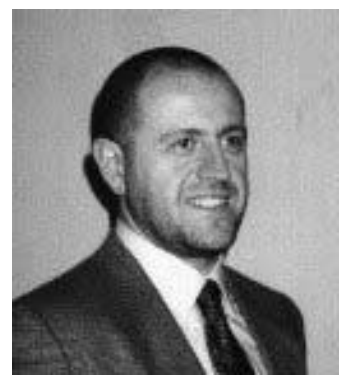

Daniele D. Giusto received the M.S. degree in Electronic Engineering in 1986, and the Ph.D. degree in Telecommunications in 1990, both from the University of Genoa, Italy. Since 1994, he is a permanent member of Faculty of Engineering, University of Cagliari, Italy, where he was appointed Full Professor of Telecommunications in 2002. In 1995 and 1998, he was a visiting professor at the Institute for Telecommunications, Technical University of Braunschweig, Germany.

He is the recipient of the 1993 AEI Ottavio Bonazzi best paper award and co-recipient of a 1998 IEEE Chester Sall best paper award. He is also a member of the executive board of CNIT, the Italian University Consortium for Telecommunications. Since 1999 , he is acting as head of Italian delegation within the ISO-JPEG international standardization committee.

$\mathrm{He}$ as been Guest Editor for some journals, acted as General Chair for the PACKET VIDEO 2000 International Workshop and the 1st International Workshop on JPEG2000. His research interests are in the area of image and video processing and coding, multimedia systems, digital television, and personal communications. Dr. Giusto is a senior member of IEEE. 